\title{
Semi-Replication-Competent Retroviral Vectors Expressing Gibbon Ape Leukemia Virus Fusogenic Membrane Glycoprotein (GALV FMG) Gene for Cancer Gene Therapy
}

\author{
Byoung Kwon Kang, Yong-Tae Jung* \\ Department of Microbiology, Dankook University, Cheonan 31116, Republic of Korea
}

\author{
Corresponding \\ Yong-Tae Jung, Professor \\ Department of Microbiology, Dankook \\ University, Cheonan 31116, Republic of \\ Korea \\ Phone : +82-41-550-3453 \\ Fax : +82-41-559-7857 \\ E-mail : yjung@dankook.ac.kr
}

Received : November 25, 2020

Revised : December 17, 2020

Accepted : December 18, 2020

No potential conflict of interest relevant to this article was reported.

Copyright (C) 2020 Journal of Bacteriology and Virology

(C) This is an Open Access article distributed under the terms of the Creative Commons Attribution Non-Commercial License

(http://creativecommons.org/ license/by-nc/3.0/).
A semi-replication-competent retroviral (s-RCR) vector system in which the gag-pol and env (GALV FMG, gibbon ape leukemia virus fusogenic membrane glycoprotein) genes were split into two separate packageable vectors was developed. These vectors are more efficient than replication-defective retroviral (RDR) vectors in gene delivery and have a higher transgene capacity than replication-competent retroviral $(R C R)$ vectors. For the gag-po/ vector construction, internal ribosomal entry site-enhanced green fluorescent protein (IRES-EGFP) was introduced downstream of the gag-po/ sequence of the previously constructed MoMLV-10A1-EGFP vector to generate MoMLV-gag-po/IRES-EGFP. For env vector construction, GALV FMG was inserted into the $p C L X S N$ vector to generate $p C L X S N-G A L V$ FMG-IRES-EGFP. MoMLV-gag-po/IRES-EGFP and pCLXSN-GALV FMG-IRES-EGFP were co-transfected into 293T cells to generate s-RCR viruses. These viruses propagated EGFP and induced syncytium formation due to the cytotoxicity of GALV FMG. To improve the cytotoxicity of s-RCR vector system, GALV FMG or the fusogenic envelope $G$ glycoprotein of the vesicular stomatitis virus (VSV-G) was inserted into gag-pol vector. Co-transfection of MoMLV-gag-po/IRES-GALV FMG + MoMLV-EGFP or MoMLV-VSV-G + pCLXSN-GALV FMG-IRES-EGFP in 293T cells induced stronger syncytium formation than s-RCR vectors (MoMLV-gag-po/IRES-EGFP + pCLXSN-GALV FMG-IRES-EGFP). In addition, s-RCR stocks collected from transfected 293T cells induced syncytium formation in the human cancer cell lines HT1080 and TE671. Hence, the s-RCR vector systems developed in this study are useful tools for cancer gene therapy.

Key Words: Cancer gene therapy, Fusogenic envelope glycoprotein, GALV FMG, $\mathrm{s}$-RCR vector, Syncytium

\section{INTRODUCTION}

Replication-defective retroviral (RDR) vectors have insufficient gene transfer efficacy and carry the risk of insertional mutagenesis. To improve the low-level transduction efficiency of these vectors, a replication-competent retroviral (RCR) vector system based on the full-length molecular clones of Moloney murine leukemia virus (MoMLV) was developed (1-7). RCR vectors have demonstrated improved efficacy of gene delivery, however, they still present the risk of accidental spread. To mitigate this risk, semi-replication competent retroviral (s-RCR) vectors were developed ( 8 , 9). s-RCR vectors are based on two transcomplementing RDR vectors (gag-po/and 
env vector). They propagate transgenes as efficiently as RCR vectors and have an insert capacity of up to $7.3 \mathrm{~kb}$ of transgene, compared to $1.3 \mathrm{~kb}$ for RCR vectors. The higher capacity of s-RCR vectors allows for the insertion of additional therapeutic transgenes.

Fusogenic membrane glycoproteins (FMGs) are viral envelope proteins which induce tumor cell killing through syncytium formation (10-13). In previous studies, the cytotoxicity of FMGs from human immunodeficiency virus (HIV), human endogenous retrovirus-W (HERV-W), vesicular stomatitis virus G glycoprotein (VSV-G), F and H genes of measles virus, feline endogenous virus RD-114, and gibbon ape leukemia virus (GALV) were evaluated (14-16). It is known that the cytotoxic activity of the R peptide truncated GALV envelope glycoprotein (GALV FMG) is higher than that of any other FMG $(11,15)$. FMG-mediated syncytium formation requires the presence of relevant cell surface receptor, and the induction of syncytia by some MLVs depend on receptor density (17). In addition, the bystander effect of FMG-mediated tumor cell killing is one log higher than that of suicide genes such as herpes simplex virus-1 thymidine kinase/ (HSV-1tk)/ Ganciclovir (GCV) or cytosine deaminase (CD)/5-fluorocytosine $(5-F C)(10,18)$.

Trajcevski et al. reported that s-RCR vectors pseudotyped with amphotropic MLV 4070A envelope allow propagations as efficient as those of RCR vector. In studies using MLV-sensitive mice, the S-RCR vector did not spread to other sites and showed no leukemia (9). Other researchers have successfully constructed s-RCR vectors pseudotyped with VSV-G (8). They incorporated VSV-G into the env vector to see if it could serve both as a pseudotyping envelope and a cytotoxic protein. s-RCR vectors pseudotyped with VSV-G induced both viral spread and progressive syncytial formation. However, VSV-G protein caused membrane fusion only at low pH (19-21). Therefore, in this study, we have attempted to use GALV FMG, which causes membrane fusion at neutral $\mathrm{pH}$.

GALV FMG is a C-terminally truncated form of GALV envelope glycoprotein, from which the 16 amino acid R peptides have been deleted. The $\mathrm{R}$ peptides are cleaved by viral proteases during virion maturation, inhibiting the fusion of the envelope until the virus leaves the cell. Previous studies have reported that MoMLV lacking R peptide could induce cell-to-cell fusion in NIH3T3 cells (22). MoMLV does not induce cell-to-cell fusion in human cells, but GALV FMG is highly fusogenic to receptor expressing human cells. GALV infects human cells through the Pit1 (SLC20A1) mammalian type III inorganic phosphate transporter, which is ubiquitously expressed on human cancer cells (23-25).

To take advantage of the therapeutic potential of GALV FMG, we developed the s-RCR vector system in which the gag-pol and GALV FMG genes are split into two vectors. We showed that during viral spread, s-RCR vectors induced cancer cell killing by virtue of the cytotoxicity of GALV FMG. Our data demonstrate that the s-RCR vectors encoding GALV FMG could play an important role in cancer gene therapy.

\section{MATERIALS AND METHODS}

\section{Cell lines}

The 293T human embryonic kidney cells (ATCC CRL-11268), PG13/LNc8 cells (ATCC CRL-10685), HT1080 human fibrosarcoma cells (ATCC CCL-121), and TE671 human rhabdomyosarcoma cells (ATCC CRL-8805) were maintained in Dulbecco's modified Eagle medium (DMEM) supplemented with 10\% fetal calf serum (FCS), $100 \mathrm{U} / \mathrm{mL}$ penicillin, and 100 $\mu \mathrm{g} / \mathrm{mL}$ streptomycin.

\section{Construction of s-RCR vectors}

To obtain GALV env and GALV FMG, total DNA was extracted from PG13/LNc8 cells with G-spin ${ }^{\text {TM }}$ Total DNA Extraction Mini Kit (iNtRON biotechnology, Seoul, Korea) according to the manufacturer's instructions. The sequences of primers are shown below: GALV F: 5'-AGATCTGCCACCATGGTATTGCTGCCTGGGTCCATG-3' (Bgll restriction site is underlined), GALV R: 5'-CTCGAGTTAAAGGTTACCTTCGTTCTCTAGG-3' (Xhol restriction site is underlined), FMG F: 5'-AGATCTGCCACCATGGT 
ATTGCTGCCTGGGTCCATG-3' (Bg 1 l restriction site is underlined), FMG R: 5'-CTCGAGTTACAGAATTTTAACTGCACTTATCCT' (Xhol restriction site is underlined). Polymerase chain reaction (PCR) products of the GALV env and GALV FMG were ligated into the pGEM ${ }^{\circledR}$-T Easy Vector System (Promega, Madison, WI, USA). pGEM-T-GALV env and pGEM-T-GALV FMG were digested with the restriction enzymes ECORI and Xhol; the digested fragments were ligated into previously constructed pCLXSN-IRES-EGFP to generate pCLXSN-GALV-IRES-EGFP and pCLXSN-GALV FMG-IRES-EGFP. To create MoMLV-gag-po/IRES-EGFP, previously developed RCR vector (MoMLV-10A1-EGFP) was digested with restriction enzyme EcoRl, and then self-ligated to delete env gene. Construction of plasmids MoMLV-VSV-G and MoMLV-EGFP has been described previously (26). To determine the viral titers, GFP-positive cells were analyzed by FACSCaliburTM flow cytometer (Becton, Dickinson and Company, Franklin Lakes, NJ USA).

\section{S-RCR virus production}

To generate s-RCR vectors encoding GALV FMG, 293T cells were transiently co-transfected with MoMLV-gag-pot IRES-EGFP, alongside either pCLXSN-GALV-IRES-EGFP or pCLXSN-GALV FMG-IRES-EGFP, using the CalPhos Mammalian Transfection Kit (TaKaRa Bio, Shiga, Japan). To investigate whether the cytotoxicity of GALV FMG could be enhanced by the addition of VSV-G, pCLXSN-GALV FMG-IRES-EGFP and MoMLV-VSV-G were co-transfected to generate VSV-G pseudotyped s-RCR vectors. One milliliter aliquots of viral supernatants at 6 days post-transfection of 293T cells were added to TE671 and HT1080 cells in the presence of $8 \mu \mathrm{g} / \mathrm{ml}$ polybrene.

\section{Construction of gag-po/ vector encoding GALV FMG}

To check if the degree of syncytium formation is dependent on whether GALV FMG is located on the gag-po/ or the env vector, MoMLV-gag-po/IRES-GALV FMG was constructed by replacing EGFP of MoMLV-gag-po/IRES-EGFP with GALV FMG. To produce gag-pol vector containing GALV FMG, MoMLV-gag-po/IRES-GALV FMG and MoMLV-EGFP were co-transfected into 293T cells. For the production of env vector containing GALV FMG, pCLXSN-GALV FMG-IRES-EGFP and MoMLV-gag-po/IRES-EGFP were co-transfected into 293 T cells. To determine the degree of syncytium formation, HT1080 cells were infected with $1 \mathrm{ml}$ of supernatants collected at 6 days post-transfection. The syncytium formation by GALV FMG was observed using fluorescence microscopy. The number of nuclei per syncytium counted from three random fields per well by microscopic examination. Results were reported as the average of two independent experiments.

\section{RESULTS}

\section{Generation of the s-RCR vector system}

To produce gag-po/vector carrying EGFP reporter transgene, MoMLV-gag-po/IRES-EGFP was constructed from replicationcompetent MoMLV-10A1-IRES-EGFP recombinant vector by deleting 10A1 env gene (Fig. 1). To create the env vector ( $p C L X S N-G A L V$ FMG-IRES-EGFP), we inserted GALV FMG into retroviral vector pCLXSN, with an IRES-EFGP positioned directly downstream of GALV FMG. To obtain high titers of viral stocks, previously constructed MoMLV-VSV-G was used to produce s-RCR vectors. MoMLV-VSV-G was generated by positioning VSV-G directly downstream of the gag-pol of MoMLV-gag-po/IRES-EGFP (26). To test whether gag-po/ could increase syncytium formation of GALV FMG, all-in-one MoMLV-gag-po/IRES-GALV FMG was also constructed.

\section{Production of s-RCR virus}

To produce s-RCR viruses, MoMLV-gag-po/IRES-EGFP with either pCLXSN-GALV FMG-IRES-EGFP or pCLXSN-GALV-IRES-EGFP was co-transfected into 293T cells. We observed a progressive increase, both in the percentage of EGFP-positive cells and as well as syncytium formation, at 2 days post-transfection. The viral titers from 293T cells transiently transfected with 


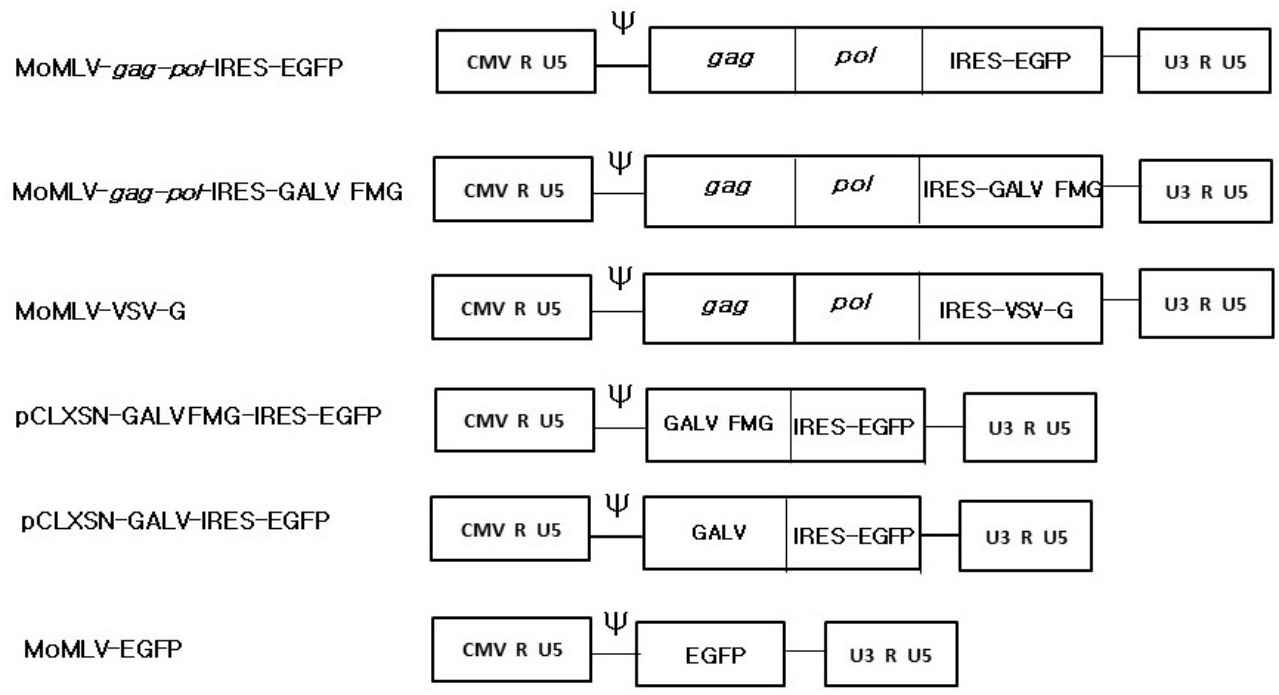

Fig. 1. Schematic structure of semi-replication-competent retroviral vectors. All vectors were derived from the full-length molecular clone of MoMLV. The MoMLV-gag-po/IRES-EGFP vector contains an IRES-EGFP cassette between the po/gene and the 3' UTR. The MoMLV-gag-po/IRES-GALV FMG is a replication-defective vector that contains the 16-amino acid R-peptide-truncated GALV env (GALV FMG). For the MoMLV-VSV-G, EGFP of the MoMLV-gag-po/ARES-EGFP was replaced with the fusogenic envelope $G$ glycoprotein of the vesicular stomatitis virus (VSV-G). The pCLXSN-GALV FMG-IRES-EGFP was generated by cloning GALV FMG into pCLXSN retroviral vector. pCLXSN-GALV-IRES-EGFP was generated by replacing GALV FMG of pCLXSN-GALV FMG-IRES-EGFP with GALV env. In MoMLV-EGFP, EGFP was inserted into pCLXSN retroviral vector.

MoMLV-gag-po/IRES-EGFP and pCLXSN-GALV FMG-IRES-EGFP at 2 days post-transfection were $3 \times 10^{6}$ transducing unit $(\mathrm{TU}) / \mathrm{ml}$. These titers were a little higher than those obtained with MoMLV-gag-po/IRES-EGFP and pCLXSN-GALV-IRES-EGFP $\left(2 \times 10^{6} \mathrm{TU} / \mathrm{ml}\right)$. This result is consistent with previous reports that truncation of the GALV R peptide increases the incorporation of envelope protein into infectious viral particles $(27,28)$.

s-RCR from MoMLV-gag-po/IRES-EGFP and pCLXSN-GALV-IRES-EGFP showed reduced viral spread upon passage of the transfected cells (Fig. 2). In contrast, s-RCR from MoMLV-gag-po/IRES-EGFP and pCLXSN-GALV FMG-IRES-EGFP showed subsequent EGFP propagation and progressive syncytium formation. When flow cytometry analysis of EGFP expression was performed at 11 days post-transfection, cells transfected with GALV FMG showed almost 80\% EGFP-positive, while cells transfected with GALV env showed about 30\% EGFP-positive (Fig. 3).

\section{Construction of VSV-G pseudotyped S-RCR vector for cancer gene therapy}

To obtain the synergistic effect of cytotoxicity due to co-expression of VSV-G and GALV FMG, we incorporated the VSV-G into gag-pol vector. When pCLXSN-GALV FMG-IRES-EGFP alone was transfected into 293T cells, limited syncytium formation was observed at 2-6 days (Fig. 4A). In contrast, when MoMLV-VSV-G + pCLXSN-GALV FMG-IRES-EGFP were co-transfected into 293T cells, extensive syncytium formation and subsequent cell death were observed at 9 days post-transfection (Fig. 4B). Although, progressively developing toxicity was also observed in cells transfected with MoMLV-VSV-G alone (Fig. 4C), co-expression of VSV-G and GALV FMG induced extensive syncytium formation.

When TE671 and HT1080 cells were infected with the s-RCR viruses collected from MoMLV-VSV-G + pCLXSN-GALV FMG-IRES-EGFP co-transfected 293T cells at an MOI of 5, extensive syncytium formation was observed in HT1080 cells at 3 days post-transfection (Fig. 5B). In contrast, syncytium formation occurred to a lesser extent in TE671 compared to HT1080 cells (Fig. 5A). The number of nuclei per syncytia was an average of $>50$ in the HT1080 cells, while an average of \pm 20 nuclei per syncytia were detected in the TE671 cells. 


\section{Co-expression of GALV FMG in gag-po/ vector enhance syncytium formation}

The s-RCR vector system is based on gag-po/and env vectors. Previous studies have suggested that gag-po/vector spreads faster than env vector in the s-RCR vector system (9). To test whether gag-po/vector containing GALV FMG enhances syncytium formation, we constructed all-in-one MoMLV-gag-po/-IRES-GALV FMG. When 293T cells were transfected with either MoMLV-gag-po/IRES-GALV FMG + MoMLV-EGFP or MoMLV-gag-po/IRES-EGFP + pCLXSN-GALV FMG-IRES-EGFP, stronger syncytium formation was achieved in $g a g-p o /$ vectors than in env vectors. To confirm that $s-R C R$ viruses were released from transfected cells, these viruses were used to infect HT1080 (Fig. 6) at an MOI of 5. Although s-RCR viruses from MoMLV-gag-po/IRES-EGFP + pCLXSN-GALV FMG-IRES-EGFP also induced syncytium formation, it was more extensive in s-RCR viruses from MoMLV-gag-po/IRES-GALV FMG + MoMLV-EGFP. MoMLV-gag-po/-IRES-GALV FMG inducted an average of \pm 40 nuclei, but pCLXSN-GALV FMG-IRES-EGFP-mediated syncytia contained an average of \pm 25 nuclei.

Day 1

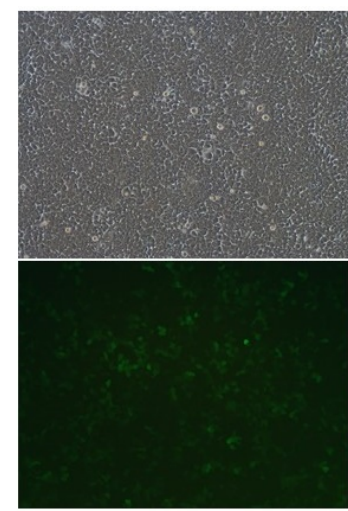

passage

Day 2 (Day 6)

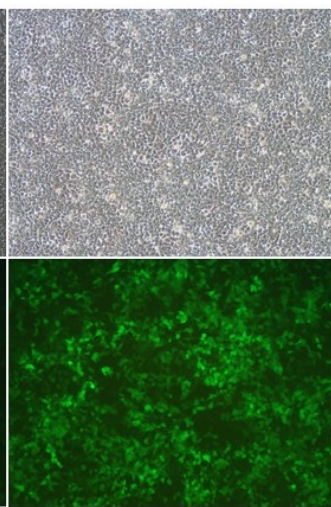

Day 9

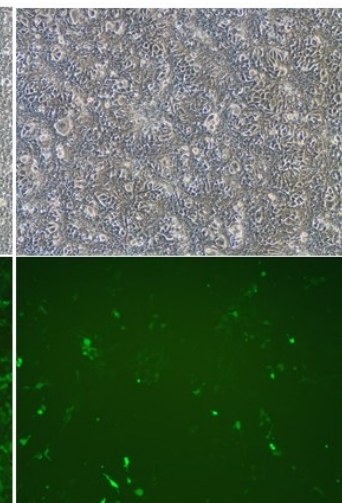

Day 11

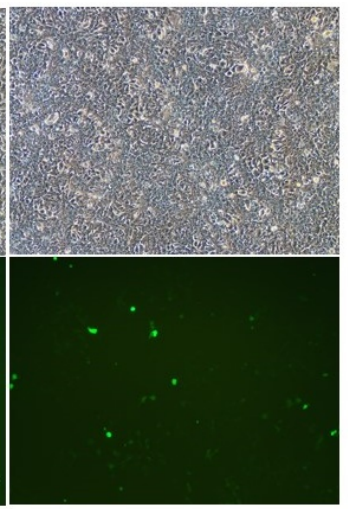

Fig. 2. In vitro EGFP gene propagation by semi-replication-competent retroviral vectors. 293T cells were co-transfected with MoMLV-gag-po/IRES-EGFP and pCLXSN-GALV-IRES-EGFP. Cells were passaged on day 6 when they became confluent. At each different time-point (Day 1, 2, 9 and 11), pictures were taken. Syncytium formation was observed using phase contrast microscopy (top panel) or fluorescence microscopy. Objective magnification, X100.

Day 1

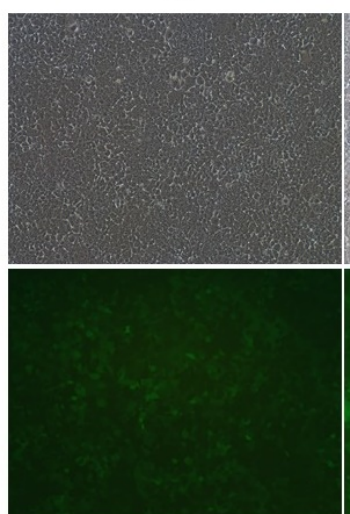

passage

(Day 6)

Day 2

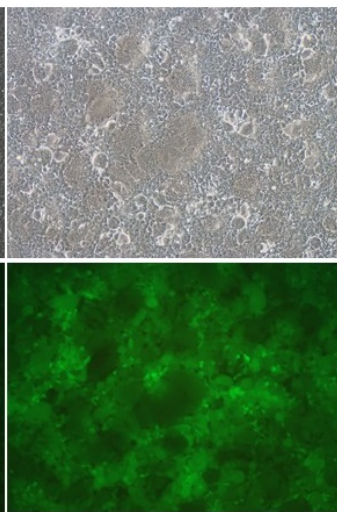

Day 9

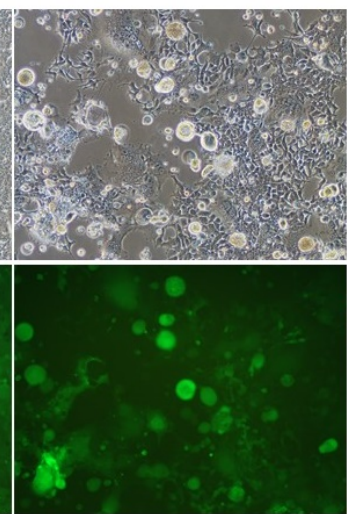

Day 11

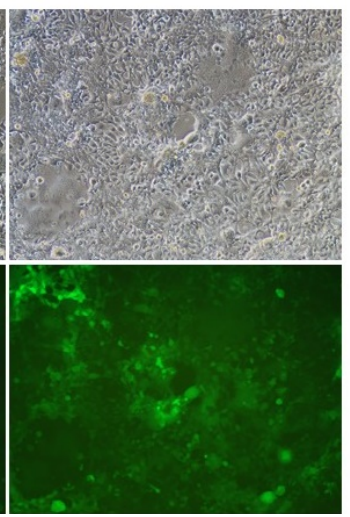

Fig. 3. In vitro EGFP gene propagation by semi-replication-competent retroviral vectors. 293T cells were co-transfected with MoMLV-gag-po/IRES-EGFP and pCLXSN-GALV FMG-IRES-EGFP. Cells were passaged on day 6 when they became confluent. At each different time-point (Day 1, 2, 9 and 11), pictures were taken. Syncytium formation was observed using phase contrast microscopy (top panel) or fluorescence microscopy. Objective magnification, X100. 


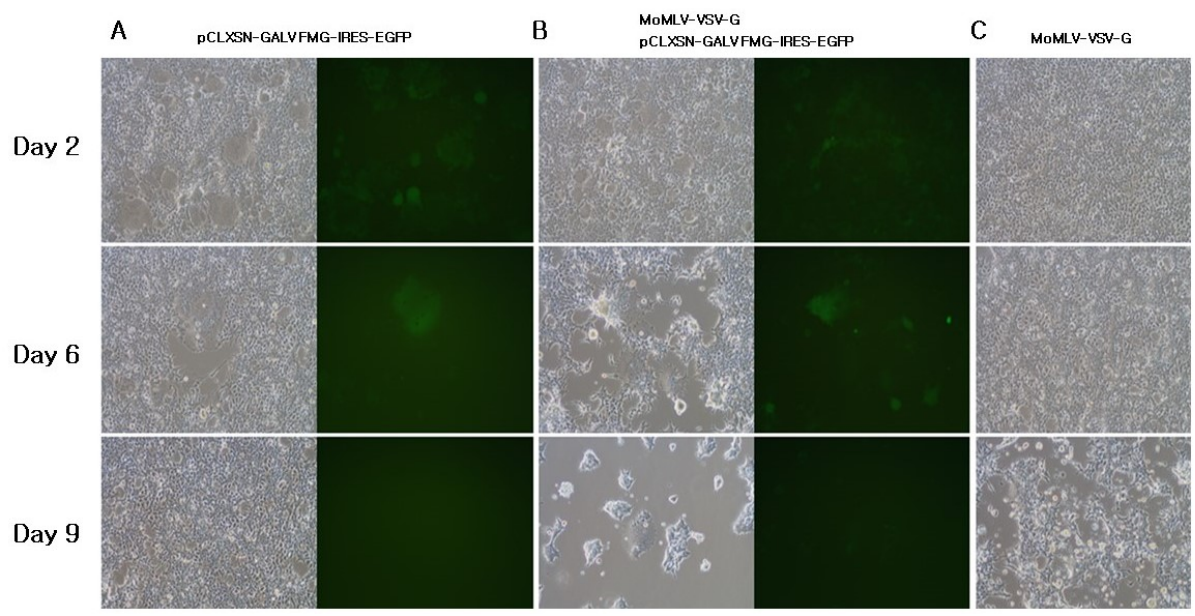

Fig. 4. Characterization of VSV-G pseudotyped s-RCR vector. 293T cells were transfected with $p C L X S N-G A L V$ FMG-IRES-EGFP (A), MoMLV-VSV-G + pCLXSN-GALV FMG-IRES-EGFP (B), MoMLV-VSV-G (C). At each different time-point (Day 2, 6 and 9), syncytium formation was observed using phase contrast microscopy or fluorescence microscopy. Objective magnification, X100.

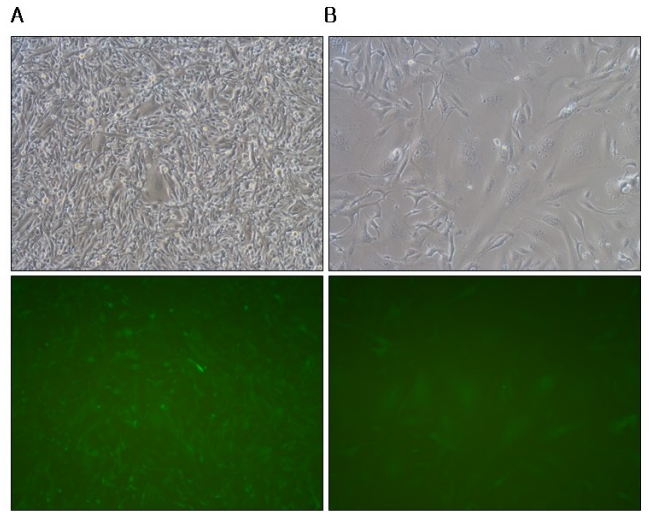

Fig. 5. Syncytium formation on human cancer cells by VSV-G pseudotyped s-RCR vector. (A) TE671 cells and HT1080 cells (B) were infected with the supernatants collected from MoMLV-VSV-G + pCLXSN-GALV FMG-IRES-EGFP co-transfected 293T cells at an $\mathrm{MOI}$ of 5 . Syncytium formation was observed at $72 \mathrm{~h}$ after infection using phase contrast microscopy (top panel) or fluorescence microscopy. Objective magnification, X200. Number of nuclei per syncytia counted from three independent experiments.
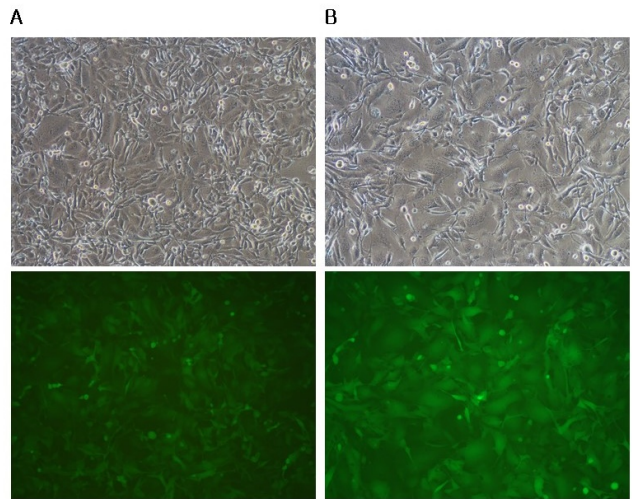

Fig. 6. Expression of GALV FMG in the gag-po/vector enhance syncytium formation. HT1080 cells were infected with the supernatants collected from MoMLV-gag-po/IRES-EGFP + pCLXSN-GALV FMG-IRES-EGFP (A) and MoMLV-gag-po/IRES-GALV FMG + MoMLV-EGFP (B) co-transfected 293T cells at an MOI of 5. Syncytium formation was observed at $72 \mathrm{~h}$ after infection using phase contrast microscopy (top panel) or fluorescence microscopy. Objective magnification, X100. Number of nuclei per syncytia counted from three independent experiments. 


\section{DISCUSSION}

Previous studies have reported the development of a s-RCR vector system using two complementary RDR vectors $(8,9)$. These vectors are safer than RCR vectors and more efficient in transgene propagation compared to RDR vectors. Coupled with their higher insert capacity, which allows for the insertion of large therapeutic transgenes, they have distinct advantages over the RCR vectors, the latter being unstable at insert sizes of $1.6 \mathrm{~kb}$ or more $(29,30)$. In this study, s-RCR vectors derived from GALV FMG were developed for cancer gene therapy (Fig. 1).

As shown in Figs. 2 and 3, an increased proportion of EGFP-positive cells is observed after s-RCR vector transfection. Interestingly, when transfected cells were passaged at day 6 , the percentage of pCLXSN-GALV FMG-IRES-EGFP-transduced cells remained stable (almost $80 \%$ of GFP positive), whereas the percentage of pCLXSN-GALV-IRES-EGFP-transduced cells was reduced (30\% of GFP positive). These interesting results suggest that syncytium can enhance cell-to-cell spread of s-RCR vector encoding GALV FMG. Previous studies have also reported that the expression of FMG in cancer cells could enhance the therapeutic efficacy of an oncolytic adenovirus and herpes simplex virus $(12,13)$. However, since cell-to-cell spread can occur in a variety of ways $(31,32)$, further studies are needed to reveal the exact mechanism of the cell-to-cell spread of s-RCR produced from MoMLV-gag-po/IRES-EGFP + pCLXSN-GALV FMG-IRES-EGFP transfected 293T cells.

Generally, VSV-G is used to make retroviral pseudotype since it can induce efficient transduction of several cell types (33). In addition, retroviruses pseudotyped with VSV-G can be concentrated by ultracentrifugation. In this study, whether VSV-G acts as a pseudotyping envelope and can show synergistic cytotoxicity with GALV FMG, previously constructed MoMLV-VSV-G was used to produce of s-RCR vectors. As shown in Fig. 4B, co-transfection of MoMLV-VSV-G + pCLXSN-GALV FMG-IRES-EGFP induced massive syncytium formation at day 9. As expected, cytotoxicity due to GALV FMG and VSV-G was stronger than that of VSV-G alone (Fig. 4C). Although both GALV FMG and VSV-G can act as pseudotyping envelopes, the latter seems to increase the production of infectious s-RCR viruses. This is because VSV-G has been reported to produce a higher titer of pseudotype retroviruses than other retroviral envelope proteins. This result agrees with a previous study which reported that the co-expression of GALV FMG and VSV-G avoids envelope mixing (18). As shown in Fig. 5, s-RCR viruses collected from transfected 293T cells induced massive syncytium formation in HT1080 compared to those from TE671. These results suggest that HT1080 cells express more receptors for GALV than TE671. In addition, since VSV-G is known to require acid shock to induce fusion in HT1080, it is believed that the syncytium formation shown in Fig. 5 is due to GALV FMG.

Previous studies have shown that gag-po/ could increase syncytium formation in truncated ecotropic envelope-expressing NIH3T3 cells (22). We also evaluated whether the presence of GALV FMG in the gag-po/vector could affect the degree of syncytium formation. s-RCR viruses produced from all-in-one MoMLV-gag-po/IRES-GALV FMG + MoMLV-EGFP induced extensive syncytium formation, whereas a much lower level of syncytium formation was observed when GALV FMG was present in env vector (Fig. 6). It is believed that this occurs because the degree of virus spread from all-in-one MoMLV-gag-po/IRES-GALV FMG + MoMLV-EGFP is faster than that from MoMLV-gag-po/IRES-EGFP + pCLXSN-GALV FMG-IRES-EGFP vector. Indeed, pCLXSN-GALV FMG-IRES-EGFP vector-transduced cells blocks re-infection by gag-pol vector using the same envelope. This interference phenomenon probably led to lower level of syncytium formation. Additionally, our results suggest that the all-in-one MoMLV-gag-po/IRES-GALV FMG vector can act as a replicationcompetent vector, just like the previously constructed all-in-one MoMLV-VSV-G (26).

In this study, s-RCR vectors encoding GALV FMG were constructed, which induced syncytium formation due to the cytotoxicity of GALV FMG. In addition, we inserted GALV FMG or the fusogenic envelope G glycoprotein of the VSV into $g a g-p o / v e c t o r$ to improve the cytotoxicity of s-RCR vector system. These novel s-RCR vectors significantly enhanced syncytium formation in transfected as well as infected cancer cell lines. Hence, these s-RCR vector systems are useful tools for cancer treatment. 


\section{ACKNOWLEDGEMENTS}

The present research was supported by the research fund of Dankook university in 2018.

\section{REFERENCES}

1) Hiraoka K, Kimura T, Logg CR, Tai CK, Haga K, Lawson GW, et al. Therapeutic efficacy of replication-competent retrovirus vector-mediated suicide gene therapy in a multifocal colorectal cancer metastasis model. Cancer Res 2007:67:5345-53.

2) Kubo S, Takagi-Kimura M, Logg CR, Kasahara N. Highly efficient tumor transduction and antitumor efficacy in experimental human malignant mesothelioma using replicating gibbon ape leukemia virus. Cancer Gene Ther 2013;20:671-7.

3) Kubo S, Takagi-Kimura M, Kasahara N. Efficient tumor transduction and antitumor efficacy in experimental human osteosarcoma using retroviral replicating vectors. Cancer Gene Ther 2019;26:41-7.

4) Lu YC, Chen YJ, Yu YR, Lai YH, Cheng JC, Li YF, et al. Replicating retroviral vectors for oncolytic virotherapy of experimental hepatocellular carcinoma. Oncol Rep 2012:28:21-6.

5) Solly SK, Trajcevski S, Frisén C, Holzer GW, Nelson E, Clerc B, et al. Replicative retroviral vectors for cancer gene therapy. Cancer Gene Ther 2003:10:30-9.

6) Song JJ, Kim JH, Lee H, Kim E, Kim J, Park YS, et al. Enhancement of gene transfer efficiency into human cancer cells by modification of retroviral vectors and addition of chemicals. Oncol Rep 2000;7:119-24.

7) Tai CK, Wang WJ, Chen TC, Kasahara N. Single-shot, multicycle suicide gene therapy by replication-competent retrovirus vectors achieves long-term survival benefit in experimental glioma. Mol Ther 2005:12:842-51.

8) Qiao J, Moreno J, Sanchez-Perez L, Kottke T, Thompson J, Caruso M, et al. VSV-G pseudotyped, MuLV-based, semi-replication-competent retrovirus for cancer treatment. Gene Ther 2006:13:1457-70.

9) Trajcevski S, Solly SK, Frisén C, Trenado A, Cosset FL, Klatzmann D. Characterization of a semi-replicative gene delivery system allowing propagation of complementary defective retroviral vectors. J Gene Med 2005;7:276-87.

10) Bateman A, Bullough F, Murphy S, Emiliusen L, Lavillette $D$, Cosset FL, et al. Fusogenic membrane glycoproteins as a novel class of genes for the local and immune-mediated control of tumor growth. Cancer Res 2000;60:1492-7.

11) Fielding AK, Chapel-Fernandes S, Chadwick MP, Bullough FJ, Cosset FL, Russell SJ. A hyperfusogenic gibbon ape leukemia envelope glycoprotein: targeting of a cytotoxic gene by ligand display. Hum Gene Ther 2000;11:817-26.

12) Fu X, Tao L, Jin A, Vile R, Brenner MK, Zhang X. Expression of a fusogenic membrane glycoprotein by an oncolytic herpes simplex virus potentiates the viral antitumor effect. Mol Ther 2003;7:748-54.

13) Guedan S, Grases D, Rojas JJ, Gros A, Vilardell F, Vile R, et al. GALV expression enhances the therapeutic efficacy of an oncolytic adenovirus by inducing cell fusion and enhancing virus distribution. Gene Ther 2012:19:1048-57.

14) Galanis E, Bateman A, Johnson $K$, Diaz RM, James CD, Vile R, et al. Use of viral fusogenic membrane glycoproteins as novel therapeutic transgenes in gliomas. Hum Gene Ther 2001;12:811-21.

15) Lin EH, Salon C, Brambilla E, Lavillette D, Szecsi J, Cosset FL, et al. Fusogenic membrane glycoproteins induce syncytia formation and death in vitro and in vivo: a potential therapy agent for lung cancer. Cancer Gene Ther 2010:17:256-65. 
16) Zhang J, Frolov I, Russell SJ. Gene therapy for malignant glioma using Sindbis vectors expressing a fusogenic membrane glycoprotein. J Gene Med 2004;6:1082-91.

17) Chung M, Kizhatil K, Albritton LM, Gaulton GN. Induction of syncytia by neuropathogenic murine leukemia viruses depends on receptor density, host cell determinants, and the intrinsic fusion potential of envelope protein. $J$ Virol 1999:73:9377-85.

18) Diaz RM, Bateman A, Emiliusen L, Fielding A, Trono D, Russell SJ, et al. A lentiviral vector expressing a fusogenic glycoprotein for cancer gene therapy. Gene Ther 2000;7:1656-63.

19) Ferlin A, Raux H, Baquero E, Lepault J, Gaudin $Y$. Characterization of pH-sensitive molecular switches that trigger the structural transition of vesicular stomatitis virus glycoprotein from the postfusion state toward the prefusion state. $J$ Viro/2014:88:13396-409.

20) Rücker $P$, Wieninger SA, Ullmann GM, Sticht $H$. pH-dependent molecular dynamics of vesicular stomatitis virus glycoprotein G. Proteins 2012:80:2601-13.

21) Yao Y, Ghosh K, Epand RF, Epand RM, Ghosh HP. Membrane fusion activity of vesicular stomatitis virus glycoprotein $\mathrm{G}$ is induced by low pH but not by heat or denaturant. Virology 2003:310:319-32.

22) Ragheb JA, Anderson WF. pH-independent murine leukemia virus ecotropic envelope-mediated cell fusion: implications for the role of the R peptide and p12E TM in viral entry. J Viro/ 1994;68:3220-31

23) Kavanaugh MP, Miller DG, Zhang W, Law W, Kozak SL, Kabat D, et al. Cell-surface receptors for gibbon ape leukemia virus and amphotropic murine retrovirus are inducible sodium-dependent phosphate symporters. Proc Natl Acad Sci U S A 1994;91:7071-5.

24) Lam JS, Reeves ME, Cowherd R, Rosenberg SA, Hwu P. Improved gene transfer into human lymphocytes using retroviruses with the gibbon ape leukemia virus envelope. Hum Gene Ther 1996;7:1415-22.

25) Miller AD. Cell-surface receptors for retroviruses and implications for gene transfer. Proc Natl Acad Sci U S A 1996:93:11407-13.

26) Jin SY, Jung YT. Construction of a replication-competent retroviral vector for expression of the VSV-G envelope glycoprotein for cancer gene therapy. Arch Viro/2020;165:1089-97.

27) Christodoulopoulos I, Cannon PM. Sequences in the cytoplasmic tail of the gibbon ape leukemia virus envelope protein that prevent its incorporation into lentivirus vectors. J Viro/2001;75:4129-38.

28) Tomás HA, Mestre DA, Rodrigues AF, Guerreiro MR, Carrondo MJT, Coroadinha AS. Improved GaLV-TR glycoproteins to pseudotype lentiviral vectors: impact of viral protease activity in the production of LV pseudotypes. Mol Ther Methods Clin 2019;15:1-8.

29) Logg CR, Logg A, Tai CK, Cannon PM, Kasahara N. Genomic stability of murine leukemia viruses containing insertions at the Env-3' untranslated region boundary. J Viro/2001:75:6989-98.

30) Nowrouzi A, Glimm H, von Kalle C, Schmidt M. Retroviral vectors: post entry events and genomic alterations. Viruses 2011:3:429-55.

31) Mothes W, Sherer NM, Jin J, Zhong P. Virus cell-to-cell transmission. J Viro/2010;84:8360-8.

32) Sattentau QJ. Cell-to-Cell Spread of Retroviruses. Viruses 2010;2:1306-21.

33) Lee H, Song JJ, Kim E, Yun CO, Choi J, Lee B, et al. Efficient gene transfer of VSV-G pseudotyped retroviral vector to human brain tumor. Gene Ther 2001:8:268-73. 